\title{
Knot exteriors with additive Heegaard genus and Morimoto's Conjecture
}

\author{
TSUYOSHI KOBAYASHI \\ YO’AV RIECK
}

Given integers $g \geq 2, n \geq 1$ we prove that there exist a collection of knots, denoted by $\mathcal{K}_{g, n}$, fulfilling the following two conditions:

(1) For any integer $2 \leq h \leq g$, there exist infinitely many knots $K \in \mathcal{K}_{g, n}$ with $g(E(K))=h$.

(2) For any $m \leq n$, and for any collection of knots $K_{1}, \ldots, K_{m} \in \mathcal{K}_{g, n}$, the Heegaard genus is additive:

$$
g\left(E\left(\#_{i=1}^{m} K_{i}\right)\right)=\sum_{i=1}^{m} g\left(E\left(K_{i}\right)\right) .
$$

This implies the existence of counterexamples to Morimoto's Conjecture [17].

57M25; 57M27

\section{Introduction and statements of results}

Let $K_{i}(i=1,2)$ be knots in the 3 -sphere $S^{3}$, and let $K_{1} \# K_{2}$ be their connected sum. We use the notation $t(\cdot), E(\cdot)$, and $g(\cdot)$ to denote tunnel number, exterior, and Heegaard genus respectively. It is well known that the union of a tunnel system for $K_{1}$, a tunnel system for $K_{2}$ and a tunnel on a decomposing annulus for $K_{1} \# K_{2}$ forms a tunnel system for $K_{1} \# K_{2}$. Therefore:

$$
t\left(K_{1} \# K_{2}\right) \leq t\left(K_{1}\right)+t\left(K_{2}\right)+1 .
$$

Since $t(K)=g(E(K))-1$, this gives:

$$
g\left(E\left(K_{1} \# K_{2}\right)\right) \leq g\left(E\left(K_{1}\right)\right)+g\left(E\left(K_{2}\right)\right) .
$$

Given integers $g \geq 0$ and $n \geq 1$, we say that a knot $K$ in a closed orientable manifold $M$ admits a $(g, n)$ position if there exists a genus $g$ Heegaard surface $\Sigma$ for $M$, separating $M$ into the handlebodies $H_{1}$ and $H_{2}$, so that $H_{i} \cap K(i=1,2)$ consists of $n$ arcs that are simultaneously parallel into $\partial H_{i}$. We say that $K$ admits a $(g, 0)$ position if $g(E(K)) \leq g$. Note that if $K$ admits a $(g, n)$ position, then $K$ admits both a $(g, n+1)$ position and a $(g+1, n)$ position. 
From Morimoto [17, Proposition 1.3], it is known that if $K_{i}(i=1$ or 2) admits a $\left(t\left(K_{i}\right), 1\right)$ position, then Inequality (1) is strict:

$$
g\left(E\left(K_{1} \# K_{2}\right)\right)<g\left(E\left(K_{1}\right)\right)+g\left(E\left(K_{2}\right)\right) .
$$

Morimoto proved that if $K_{1}$ and $K_{2}$ are m-small knots ${ }^{1}$ in $S^{3}$, then the converse holds [17, Theorem 1.6]. This result was generalized to arbitrarily many m-small knots in general manifolds by the authors [9]. Morimoto conjectured that the converse holds in general [17, Conjecture 1.5]:

Morimoto's Conjecture Given knots $K_{1}, K_{2} \subset S^{3}$,

$$
g\left(E\left(K_{1} \# K_{2}\right)\right)<g\left(E\left(K_{1}\right)\right)+g\left(E\left(K_{2}\right)\right)
$$

if and only if $K_{i}$ admits a $\left(t\left(K_{i}\right), 1\right)$ position (for $i=1$ or $i=2$ ).

Remark 1.1 Morimoto stated the above conjecture in terms of 1-bridge genus $g_{1}(K)$. It is easy to see that Conjecture 1.5 of [17] is equivalent to the statement above.

In [10] the authors showed that the existence of a knot $K$ satisfying the two conditions below implies the existence of counterexamples to Morimoto's Conjecture:

- $K$ does not admit a $(t(K), 2)$ position.

- $K$ is m-small.

We asked [10, Question 1.9] if there exists a knot $K$ with $g(E(K))=2$ that does not admit a $(1,2)$ position; this question was answered affirmatively by Johnson and Thompson. In fact, in [5, Lemma 4] Johnson showed the existence of knots $K$ with $g(E(K))=2$ admitting Heegaard splittings with arbitrarily high distance (see Definition 2.4), and in [6, Corollary 2] Johnson and Thompson showed that (for any $n$ ) infinitely many of these knots do not admit a $(1, n)$ position. At about the same time Minsky, Moriah and Schleimer [11, Theorem 3.1] proved a more general result, showing that for any integer $g \geq 2$, there exist infinitely many knots $K$ with $g(E(K))=g$ admitting a minimal genus Heegaard splitting with arbitrarily high distance. By Proposition 2.6 (for any $n$ ) infinitely many of these knots do not admit a $(t(K), n)$ position. However, at the time of writing, the existence of an m-small knot $K$ not admitting a $(t(K), 2)$ position is not known.

\footnotetext{
${ }^{1} \mathrm{~A}$ knot $K$ is called $m$-small if its exterior does not admit an essential surface whose boundary consists of a nonempty collection of meridians of $K$.
} 
Given $n \geq 1$, consider the following conditions:

(1) $K$ does not admit a $(t(K), n)$ position.

(2) $E(K)$ does not admit an essential surface $S$ with $\chi(S) \geq 4-2 n g(E(K))$.

Our main result is Theorem 1.2 below, which implies the existence of knots fulfilling Conditions (1) and (2) for each $n \geq 1$; specifically, in the proof of Theorem 1.2 we show that some of the knots whose existence was proved in [5] and [11] fulfill these conditions. In Corollary 1.5, we show that this implies the existence of counterexamples to Morimoto's Conjecture.

Theorem 1.2 Given integers $g \geq 2$ and $n \geq 1$, let $\mathcal{K}_{g, n}$ be the set of all knots $K \subset S^{3}$ with the following three properties:

(a) $g(E(K)) \leq g$.

(b) $K$ does not admit a $(t(K), n)$ position.

(c) $E(K)$ does not admits an essential surface $S$ with $\chi(S) \geq 4-2 g n$.

Then $\mathcal{K}_{g, n}$ has the following properties:

(1) For each $h, 2 \leq h \leq g$, there exists infinitely many knots $K \in \mathcal{K}_{g, n}$ with $g(E(K))=h$.

(2) For each $m \leq n$ and for any collection of knots $K_{1}, \ldots, K_{m} \in \mathcal{K}_{g, n}$ (possibly, $K_{i}=K_{j}$ for $i \neq j$ ) we have:

$$
g\left(E\left(\#_{i=1}^{m} K_{i}\right)\right)=\sum_{i=1}^{m} g\left(E\left(K_{i}\right)\right) .
$$

Moreover, for each $g$, we have:

$$
\bigcap_{n=1}^{\infty} \mathcal{K}_{g, n}=\varnothing .
$$

Remark 1.3 The existence of knots $K_{1}, K_{2}$ with $g\left(E\left(K_{1} \# K_{2}\right)\right)=g\left(E\left(K_{1}\right)\right)+$ $g\left(E\left(K_{2}\right)\right)$ is known from Moriah and Rubinstein [15] and Morimoto, Sakuma and Yokota [18]. Theorem 1.2 is new in the following ways:

(1) It is the first time that the connected sum of more than two knots is shown to have additive Heegaard genus.

(2) The proof in [15] uses minimal surfaces in hyperbolic manifolds and in [18] quantum invariants. Our proof is purely topological. 
A knot $K \subset M$ is called admissible if $g(E(K))>g(M)$. Thus any knot $K \subset S^{3}$ is admissible. We denote the connected sum of $m$ copies of $K$ by $m K$. By [10, Theorem 1.2] for any admissible knot $K$, there exists $N$ so that if $m>N$ then $g(E(m K))<m g(E(K))$. In contrast to this, as an obvious consequence of Theorem 1.2 we have:

Corollary 1.4 Given integers $g \geq 2$ and $n \geq 1$, there exist infinitely many knots $K \subset S^{3}$ so that $g(E(K))=g$ and for any $m \leq n, g(E(m K))=m g$.

A consequence of Corollary 1.4 is:

Corollary 1.5 There exists a counterexample to Morimoto's Conjecture. Specifically, there exist knots $K_{1}, K_{2} \subset S^{3}$ such that the following two conditions hold:

(1) $K_{i}$ does not admit a $\left(t\left(K_{i}\right), 1\right)$ position $(i=1,2)$.

(2) There exists an integer $m_{0} \geq 4$ such that:

(a) $g\left(E\left(K_{1}\right)\right)=4$.

(b) $g\left(E\left(K_{2}\right)\right)=2\left(m_{0}-2\right)$.

(c) $g\left(E\left(K_{1} \# K_{2}\right)\right)<2 m_{0}$.

The argument of the proof of Corollary 1.5 was originally given in [10, Theorem 1.4]. We outline it here for completeness.

Proof of Corollary 1.5 Let $K$ be a knot as in Corollary 1.4, for $g=2$ and $n=3$. By [10, Theorem 1.2], for some $m>1, g(E(m K))<m g(E(K))=2 m$. Let $m_{0}$ be the minimal number with that property. Since we chose $K$ for $n=3, m_{0} \geq 4$. Hence $g(E(2 K))=2 g(E(K))=4$. By the minimality of $m_{0}, g\left(E\left(\left(m_{0}-2\right) K\right)\right)=$ $\left(m_{0}-2\right) g(E(K))=2\left(m_{0}-2\right)$. Let $K_{1}=2 K$ and $K_{2}=\left(m_{0}-2\right) K$. Note that $K_{1} \# K_{2}=m_{0} K$. Thus:

(a) $g\left(E\left(K_{1}\right)\right)=4$.

(b) $g\left(E\left(K_{2}\right)\right)=2\left(m_{0}-2\right)$.

(c) $g\left(E\left(K_{1} \# K_{2}\right)\right)<2 m_{0}$.

We claim that $K_{1}$ does not admit a $\left(t\left(K_{1}\right), 1\right)$ position. Assume for a contradiction it does. By Inequality (2) and the above (a), $g(E(3 K))=g\left(E\left(K_{1} \# K\right)\right)<g\left(E\left(K_{1}\right)\right)+$ $g(E(K))=6$. Since $m_{0} \geq 4, g(E(3 K))=3 g(E(K))=6$, which is a contradiction.

We claim that $K_{2}$ does not admit a $\left(t\left(K_{2}\right), 1\right)$ position. Assume for a contradiction it does. By Inequality (2) and the above (b), $g\left(E\left(\left(m_{0}-1\right) K\right)\right)<g\left(E\left(\left(m_{0}-2\right) K\right)\right)+$ $g(E(K))=\left(m_{0}-1\right) g(E(K))$. By the minimality of $m_{0}, g\left(E\left(\left(m_{0}-1\right) K\right)\right)=$ $\left(m_{0}-1\right) g(E(K))$, which is a contradiction. 
We note that $K_{1}$ and $K_{2}$ are composite knots. This led Moriah to conjecture [13, Conjecture 7.14] that if $K_{1}$ and $K_{2}$ are prime then Morimoto's Conjecture holds.

Outline Section 2 is devoted to three propositions necessary for the proof of Theorem 1.2: Proposition 2.2 relates strongly irreducible Heegaard splittings and bridge position, Proposition 2.5 relates essential surfaces and the distance of Heegaard splitting (Proposition 2.5 is exactly Theorem 3.1 of Scharlemann [22]), and Proposition 2.6 relates bridge position and distance of Heegaard splittings (Proposition 2.6 is exactly Theorem 1 of Johnson and Thompson [6] except for knots $K \subset M$ that admit a $(t(K), 1)$ position and are isotopic onto a Heegaard surface for $M$ of genus $t(K))$. In Section 3 we calculate the genera of certain manifolds that we denote by $X^{(c)}$ (see Notation 2.1). In Section 4 we prove Theorem 1.2.

Remarks 1.6 (1) Tomova, independently and using different techniques, obtained a stronger result than Proposition 2.6 [28, Theorem 1.3].

(2) We refer the reader to our paper [7], that can be used as an introduction to the ideas in the current paper. In [7] an easy argument is given for a special case of Corollary 1.4, namely, $g=2$ and $n=3$. Note that this special case is sufficient for Corollary 1.5.

\section{Decomposing $X^{(c)}$}

In this and the following sections, we adopt the following notation.

Notation 2.1 Let $K$ be a knot in a closed orientable connected manifold $M$ and $X$ its exterior. For an integer $c \geq 0$ we denote by $X^{(c)}$ the manifold obtained by drilling $c$ curves out of $X$ that are simultaneously parallel to meridians of $K$. Note that $X^{(0)}=X$.

Proposition 2.2 Let $X, X^{(c)}$ be as above and $g \geq 0$ an integer. Suppose that for some integer $c>0, X^{(c)}$ admits a strongly irreducible Heegaard surface of genus $g$. Then one of the following holds:

(1) $X$ admits an essential surface $S$ with $\chi(S) \geq 4-2 g$.

(2) (a) $c \leq g$, and

(b) for some $b, c \leq b \leq g, K$ admits a $(g-b, b)$ position. 
Proof of Proposition 2.2 Assume Conclusion (1) does not hold.

Let $C_{1} \cup_{\Sigma} C_{2}$ be a genus $g$ strongly irreducible Heegaard splitting of $X^{(c)}$. Since $c>0, X^{(c)}$ admits an essential torus $T$ that gives the decomposition $X^{(c)}=X^{\prime} \cup_{T}$ $Q^{(c)}$, where $X^{\prime} \cong X$ and $Q^{(c)}$ is a $c$-times punctured annulus cross $S^{1}$. Since $T$ is incompressible and $\Sigma$ is strongly irreducible, we may isotope $\Sigma$ so that every component of $\Sigma \cap T$ is essential in both surfaces (see, for example, Schultens [26, Lemma 6]). Isotope $\Sigma$ to minimize $|\Sigma \cap T|$ subject to this constraint. Denote $\Sigma \cap X^{\prime}$ by $\Sigma_{X}$, and $\Sigma \cap Q^{(c)}$ by $\Sigma_{Q}$. Note that, since $T$ is essential, $\Sigma \cap T \neq \varnothing$. By the minimality of $|\Sigma \cap T|$ no component of $\Sigma_{X}$ (resp. $\Sigma_{Q}$ ) is boundary parallel in $X^{\prime}$ (resp. $\left.Q^{(c)}\right)$.

We claim that $\Sigma_{X}$ is connected and compresses into both sides in $X^{\prime}$, and that $\Sigma_{Q}$ is incompressible in $Q^{(c)}$. We sketch this argument here (see [9, Claim 4.5]). By the minimality of $|\Sigma \cap T|$, for $i=1,2$, the components of $T \cap C_{i}$ are incompressible, non-boundary parallel annuli in $C_{i}$. It follows that there is a meridian disk $D_{i} \subset C_{i}$ which is disjoint from $T$. Hence there is some component of $\Sigma$ cut open along $T$ that compresses into $C_{1}$ and some component that compresses into $C_{2}$. By strong irreducibility of $\Sigma$, the same component compresses into both sides; moreover, all other components are incompressible. As remarked above no component of $\Sigma$ cut open along $T$ is boundary parallel; hence any incompressible component is essential. If some such component is in $X^{\prime}$ then Conclusion (1) holds, contradicting our assumption. Hence $\Sigma_{X}$ is connected and compresses into both sides, and every component of $\Sigma_{Q}$ is essential. This completes the proof of the claim.

Since $Q^{(c)}$ is a punctured annulus cross $S^{1}$ and $\Sigma_{Q}$ is incompressible and has no boundary parallel or closed component, every component of $\Sigma_{Q}$ is a vertical annulus (see, for example, Jaco [4, VI.34]). Hence $\partial \Sigma_{X}$ consists of meridians of $K$. For $i=1,2$, let $\Sigma_{i}$ be the surface obtained by simultaneously compressing $\Sigma_{X}$ maximally into $C_{i} \cap X^{\prime}$. (By simultaneous compression, we mean compressing $\Sigma_{X}$ once along a collection of mutually disjoint disks, without iterations.) Then the argument of Claim 6 of [8, page 248] shows that every component of $\Sigma_{i}$ is incompressible. Hence, every component of $\Sigma_{i}$ is a boundary parallel annulus in $X^{\prime}$ or a $2-$ sphere, for otherwise Conclusion (1) holds, contradicting our assumption. Denote the number of boundary parallel annuli by $b$ (note that $b=\frac{1}{2}\left|\partial \Sigma_{X}\right|$ and is the same for $\Sigma_{1}$ and $\Sigma_{2}$ ). Denote the solid tori that define the boundary parallelism of the annular components of $\Sigma_{i}$ by $N_{i, 1}, \ldots, N_{i, b}(i=1,2)$.

Claim 1 For each $i(i=1,2), N_{i, 1}, \ldots, N_{i, b}$ are mutually disjoint.

Proof of Claim 1 Assume, for a contradiction, that two components (say $N_{i, 1}$ and $\left.N_{i, 2}\right)$ intersect, say $N_{i, 2} \subset N_{i, 1}$. Note that $\Sigma_{X}$ is retrieved from $\Sigma_{i}$ by tubing. Since 
$\Sigma_{i}$ is obtained from $\Sigma_{X}$ by simultaneously compressing into the $C_{i}$ side only and $\Sigma_{X}$ is connected, all the tubes are contained in $N_{i, 1}$. This implies that $N_{i, j} \subset N_{i, 1}$ for all $j$. This shows that $\Sigma$ is isotopic into $Q^{(c)}$, hence $T$ is isotopic into $C_{1}$ or $C_{2}$. Since $T$ is essential, this is impossible. This proves Claim 1.

Remark 2.3 As a part of the proof of Proposition 2.2, we analyze the intersection of $\Sigma$ with $Q^{(c)}$. When $K$ is a hyperbolic knot, $Q^{(c)}$ is a component of the characteristic subvariety. We point the reader to [23, Theorem 3.8], where Scharlemann and Schultens treat the intersection of a strongly irreducible Heegaard surface with the characteristic subvariety in general. Our setting is more limited, and this allows us to obtain more detailed information, eg Claim 2 below.

Claim $2 K$ admits a $(g-b, b)$ position.

Proof of Claim 2 For each $i(i=1,2)$, let $A_{i, j}$ be the annulus $N_{i, j} \cap T$ ( $j=$ $1, \ldots, b)$. Note that $A_{i, j}$ is a longitudinal annulus in $N_{i, j}$. By Claim $1, C_{i} \cap X^{\prime}$ is obtained from $N_{i, 1}, \ldots, N_{i, b}$ and a (possibly empty) collection of 3-balls by attaching 1-handles. Hence $C_{i} \cap X^{\prime}$ is a handlebody and $\left\{A_{i, j}\right\}_{j=1}^{b}$ is a primitive system of annuli in $\partial\left(C_{i} \cap X^{\prime}\right)$, ie there exists a system of properly embedded disjoint disks $\left\{\Delta_{i, j}\right\}_{j=1}^{b}$ such that $\Delta_{i, j} \cap A_{i, k}=\varnothing$ for $j \neq k$, and $\Delta_{i, j} \cap A_{i, j}$ is a spanning arc for $A_{i, j}$.

Since $X^{\prime}$ is homeomorphic to $X$, we may perform the trivial Dehn filling on $X^{\prime}$ to obtain $M$. In $M$ we cap $\Sigma_{X}$ off by attaching $2 b$ disks to obtain a genus $g-b$ closed surface, say $S$. Then $S$ separates $M$ into two parts, denoted $H_{1}$ and $H_{2}$, so that $H_{i}$ is obtained from $C_{i} \cap X^{\prime}$ by attaching $b 2$-handles along $A_{i, 1}, \ldots, A_{i, b}$. Since the system $\left\{A_{i, j}\right\}_{j=1}^{b}$ is primitive, $H_{i}$ is a handlebody. Hence $H_{1} \cup_{S} H_{2}$ is a Heegaard splitting of $M$.

Up to isotopy, the knot $K$ is the core of the attached solid torus. Thus $K \cap H_{i}(i=1,2)$ is the union of the co-cores of the 2-handles, and each co-core is isotopic into $\partial H_{i}$ via one of the disks $\Delta_{i, j}$. Since the disks $\Delta_{i, j}$ are disjoint, we see that $K \cap H_{i}$ consists of $b$ simultaneously boundary parallel arcs. Hence $H_{1} \cup H_{2}$ induces a $(g-b, b)$ position of $K$. This proves Claim 2.

To complete the proof we need to show that $c \leq b \leq g$. Since $g-b \geq 0$, it is obvious that $b \leq g$ holds. Suppose, for a contradiction, that $b<c$. Note that $\Sigma_{Q}$ consists of $b$ vertical annuli that separate $Q^{(c)}$ into $b+1$ components. Note that $\partial X^{(c)}$ consists of $c+1$ tori; thus if $b<c$ then two components of $\partial Q^{(c)}$ are in the same component of $Q^{(c)}$ cut open along $\Sigma_{Q}$. It is easy to see that there is a vertical annulus connecting 
these tori, which is disjoint from $\Sigma$. Hence this annulus is contained in a compression body $C_{i}$ and connects components of $\partial C_{i} \backslash \Sigma$. This contradiction completes the proof of Proposition 2.2.

Definition 2.4 (Hempel [3]) Let $H_{1} \cup_{\Sigma} H_{2}$ be a Heegaard splitting. The distance of $\Sigma$, denoted $d(\Sigma)$, is the least integer $d$ so that there exist meridian disks $D_{i} \subset H_{i}$ $(i=1,2)$ and essential curves $\gamma_{0}, \ldots, \gamma_{d} \subset \Sigma$ so that $\gamma_{0}=\partial D_{1}, \gamma_{d}=\partial D_{2}$, and $\gamma_{i-1} \cap \gamma_{i}=\varnothing(i=1, \ldots, d)$. There are three cases where this definition does not apply: $M \cong S^{3}$ and $g(\Sigma)=0, M$ is a genus $g$ handlebody and $g(\Sigma)=g$, and $M$ is a lens space and $g(\Sigma)=1$. In the first two cases on at least one side there are no meridian disks, and in the last case there is no sequence of curves on $\Sigma$ as required in the definition. In all three cases, we define $d(\Sigma)$ to be zero.

We need two properties of knots whose exteriors admit a Heegaard splittings of high distance. The first is Theorem 3.1 of [22] (for closed surfaces this was shown by Hartshorn [2]):

Proposition 2.5 [22] Let $K$ be a knot and $d \geq 0$ an integer. Suppose $X$ admits a Heegaard splitting with distance greater than $d$. Then $X$ does not admit a connected essential surface $S$ with $\chi(S) \geq 2-d$.

Proposition 2.6 below was first stated as Theorem 4.1 of [11]. Our proof is a combination of Theorem 1 of [6] and Corollary 4.7 of [24]. The statements of Theorem 1 of [6] and of Proposition 2.6 are very similar; however, the definitions of $(p, 0)$ position used in [6] and here are distinct. In [6] $K$ is said to admit a $(p, 0)$ position $^{2}$ if and only if $K$ is isotopic into a genus $p$ Heegaard splitting. Recall that by our definition, $K$ admits a $(p, 0)$ position if and only if $g(X) \leq p$. Thus, if $p<g(X)$ and $K$ is isotopic into a genus $p$ Heegaard surface, then $K$ admits a $(p, 0)$ in the sense of [6], and does not admit a $(p, 0)$ position in our sense; note that in that case $K$ admits a $(p, 1)$ position in our sense. In all other cases, $K$ admits a $(p, q)$ position in the sense of [6] if and only if it admits a $(p, q)$ position in our sense.

Shortly after our paper was posted, Tomova proved a stronger version of Proposition 2.6 using different techniques [28, Theorem 1.3].

Proposition 2.6 Let $K \subset S^{3}$ be a knot and $p, q$ integers so that $K$ admits a $(p, q)$ position.

If $p<g(X)$ then any Heegaard splitting for $X$ has distance at most $2(p+q)$.

\footnotetext{
${ }^{2}$ The term used in [6] is " $K$ is $(p, 0)$ ", rather than " $K$ admits a $(p, 0)$ position".
} 
Proof Suppose $K$ admits a $(p, q)$ position with $p<g(X)$. By tubing the surface that gives the bridge position $r$ times $(0 \leq r \leq q)$ we obtain a $(p+r, q-r)$ position. We take $r=g(X)-p-1$; thus $p+r=g(X)-1=t(K)$. Let $n$ be the minimal number so that $K$ admits a $(t(K), n)$ position in our sense. We see that $n \leq q-r$. Since $t(K)=p+r$, this implies that $t(K)+n \leq p+q$. Hence, for the proof of Proposition 2.6, it suffices to show that any Heegaard splitting of $X$ has distance at most $2(t(K)+n)$.

Claim 1 The knot exterior $X$ admits a minimal genus Heegaard surface with distance at most $2(t(K)+n)$.

Proof of Claim 1 Let $n^{\prime}$ be the minimal integer so that $K$ admits a $\left(t(K), n^{\prime}\right)$ position according to the definition given in [6]. Assume first that $K$ is not isotopic onto any genus $t(K)$ Heegaard surface of $S^{3}$. Then $n=n^{\prime}$, and the claim then follows directly from [6, Theorem 1].

Thus we may assume that $S^{3}$ admits a genus $t(K)$ Heegaard splitting, say $H_{1} \cup_{\Sigma} H_{2}$, so that $K \subset \Sigma$, ie, $n^{\prime}=0$. On the other hand, as explained above $n=1$. We base our analysis on $[19 ; 20 ; 21]$. We perform a tiny isotopy of $K$ in $H_{2}$, pushing it off $\Sigma$. Denote the knot obtained by $\widetilde{K} \subset H_{2}$. The image of the isotopy is an annulus (say $A$ ) embedded in $H_{2}$ so that one boundary component of $A$ is $\widetilde{K}$ and the other is $K \subset \Sigma$. Let $\alpha$ be a spanning arc for $A$. Let $\widetilde{H}_{1}=H_{1} \cup N_{H_{2}}(\alpha \cup \widetilde{K})$ and let $\widetilde{H}_{2}=\operatorname{cl}\left(M \backslash \widetilde{H}_{1}\right)$. It is easy to see that $\widetilde{H}_{1}$ and $\widetilde{H}_{2}$ are handlebodies (with $\widetilde{K} \subset \widetilde{H}_{1}$ ) and therefore $\partial \widetilde{H}_{1}=\partial \widetilde{H}_{2}$ is a Heegaard surface for $S^{3}$, denoted $S_{\widetilde{K}}(\Sigma) .{ }^{3}$ Denote the exterior of $\widetilde{K}$ by $\tilde{X}$. Note that $\tilde{X} \cong X$. In [19] it was shown that $S_{\widetilde{K}}(\Sigma)$ is a Heegaard surface for $\tilde{X}$. Since $g\left(S_{\widetilde{K}}(\Sigma)\right)=g(\Sigma)+1=t(K)+1=g(\tilde{X})$, we have that $S_{\widetilde{K}}(\Sigma)$ is a minimal genus Heegaard surface for $\tilde{X}$.

We claim $d\left(S_{\widetilde{K}}(\Sigma)\right) \leq 2$. Let $\widetilde{D}_{1} \subset \widetilde{H}_{1}$ be the disk $\operatorname{cl}\left(\Sigma \backslash S_{\widetilde{K}}(\Sigma)\right)$ and let $\gamma_{0}=\partial \widetilde{D}_{1}$. Since $t(K)>0, \gamma_{0}$ is essential in $S_{\widetilde{K}}(\Sigma)$. Let $\widetilde{D}_{2} \subset \widetilde{H}_{2}$ be the disk $A \cap \widetilde{H}_{2}$ and let $\gamma_{2}$ be $\partial \widetilde{D}_{2}$. Since $\gamma_{2}$ is nonseparating it is essential in $S_{\widetilde{K}}(\Sigma)$. Let $\gamma_{1}$ be a longitude of $\partial N_{H_{2}}(\alpha \cup \widetilde{K})$ chosen so that $\gamma_{0} \cap \gamma_{1}=\varnothing$ and $\gamma_{1} \cap \gamma_{2}=\varnothing$. Then $\gamma_{1}$ is essential in $S_{\widetilde{K}}(\Sigma)$. Hence by Definition $2.4, d\left(S_{\widetilde{K}}(\Sigma)\right) \leq 2<2(t(K)+n) .{ }^{4}$

This proves Claim 1.

Claim 2 Any Heegaard surface for $X$ has distance at most $2(t(K)+n)$.

\footnotetext{
${ }^{3} S_{\widetilde{K}}(\Sigma)$ is called stabilization of $\Sigma$ along $\widetilde{K}[19$, Definition 2.1]. For a detailed description see also Subsection 4.2 of [16].

${ }^{4}$ The referee interprets the proof above as follows: first, we show that $S_{\widetilde{K}}(\Sigma)$ is so-called $\mu$-primitive, and then we show that all $\mu$-primitive Heegaard surfaces have distance at most 2.
} 
Proof of Claim 2 Let $\Sigma$ be a Heegaard surface as in Claim 1, ie, $\Sigma$ is minimal genus and $d(\Sigma) \leq 2(t(K)+n)$. Let $\tilde{\Sigma}$ be any Heegaard surface for $X$. By [24, Corollary 4.7] (with $\Sigma$ corresponding to $Q$ and $\widetilde{\Sigma}$ to $P$ ) one of the following holds:

(1) Either $\Sigma$ is isotopic $\tilde{\Sigma}$, or $\Sigma$ is obtained from $\tilde{\Sigma}$ by stabilizations or boundary stabilizations.

(2) $d(\widetilde{\Sigma}) \leq 2 g(\Sigma)$.

We treat the cases in order:

(1) Since $\Sigma$ is a minimal genus Heegaard splitting, $\Sigma$ is isotopic to $\widetilde{\Sigma}$. Therefore $d(\widetilde{\Sigma})=d(\Sigma) \leq 2(t(K)+n)$.

(2) In this case, $d(\widetilde{\Sigma}) \leq 2 g(\Sigma)=2(t(K)+1) \leq 2(t(K)+n)$.

This proves Claim 2.

Claim 2 establishes Proposition 2.6.

\section{Calculating $g\left(X^{(c)}\right)$}

For $X^{(c)}$, recall Notation 2.1. The following lemma is an easy application of the concept of stabilizing along a knot [19, Definition 2.1] that is described in the proof of Proposition 2.6.

Lemma 3.1 Let $K \subset M$ be a knot, $X$ the exterior of $K$, and $c \geq 0$ an integer. Denote the genus of $X$ by $g$. Then

$$
g\left(X^{(c)}\right) \leq g+c .
$$

Proof The proof is an induction on $c$. For $c=0$ there is nothing to prove.

Fix $c>0$. We obtain $X^{(c-1)}$ by Dehn filling a component of $\partial X^{(c)}$ and the core of the attached solid torus (say $\gamma$ ) is isotopic into $\partial X$. Any Heegaard surface for $X^{(c-1)}$ is obtained from a torus parallel to $\partial X$ and a (possibly empty) collection of tori parallel to other components of $\partial X^{(c-1)}$ by tubing. Hence $\gamma$ is isotopic onto any Heegaard surface for $X^{(c-1)}$. By stabilizing a minimal genus Heegaard surface for $X^{(c-1)}$ along $\gamma$ we obtain a Heegaard surface for $X^{(c)}$ of genus $g\left(X^{(c-1)}\right)+1$. Hence $g\left(X^{(c)}\right) \leq g\left(X^{(c-1)}\right)+1$.

By the induction hypothesis, $g\left(X^{(c-1)}\right) \leq g+(c-1)$; hence we get: $g\left(X^{(c)}\right) \leq$ $g\left(X^{(c-1)}\right)+1 \leq g+(c-1)+1=g+c$. 
Proposition 3.2 Let $M$ be a compact orientable manifold that does not admit a nonseparating surface. Let $K \subset M$ be a knot, and $X$ its exterior. Let $c \geq 0$ be an integer. Denote the genus of $X$ by $g$. Suppose that $X$ does not admit an essential surface $S$ with $\chi(S) \geq 4-2(g+c)$, and that $K$ does not admit a $(g-1, c)$ position. Then

$$
g\left(X^{(c)}\right)=g+c .
$$

Proof The proof is an induction on $c$. For $c=0$ there is nothing to prove.

Fix $c>0$ and let $\Sigma \subset X^{(c)}$ be a minimal genus Heegaard surface. It follows from the assumptions that $X$ does not admit an essential surface $S$ with $\chi(S) \geq 4-2(g+(c-1))$, and that $K$ does not admit a $(g-1, c-1)$ position; hence the induction hypothesis applies to $X^{(c-1)}$, giving that $g\left(X^{(c-1)}\right)=g+c-1$.

The proof is divided into the following two cases:

Case $1 \Sigma$ is strongly irreducible.

By Proposition 2.2 one of the following holds:

(1) $X$ admits an essential surface $S$ with $\chi(S) \geq 4-2 g\left(X^{(c)}\right)$.

(2) $c \leq g\left(X^{(c)}\right)$, and for some $b\left(c \leq b \leq g\left(X^{(c)}\right)\right), K$ admits a $\left(g\left(X^{(c)}\right)-b, b\right)$ position.

By Lemma 3.1, we have $4-2 g\left(X^{(c)}\right) \geq 4-2(g+c)$. By assumption $X$ does not admit an essential surface $S$ with $\chi(S) \geq 4-2(g+c)$, so Case 1 above cannot happen and we may assume that we are in Case 2. Since $b-c \geq 0$, we can tube the Heegaard surface giving the $\left(g\left(X^{(c)}\right)-b, b\right)$ position $b-c$ times to obtain a $\left(g\left(X^{(c)}\right)-b+(b-c), b-(b-c)\right)=\left(g\left(X^{(c)}\right)-c, c\right)$ position.

By assumption $K$ does not admit a $(g-1, c)$ position; this implies that if $K$ admits a $(p, c)$ position for some $p$, then $p>g-1$. Thus $g\left(X^{(c)}\right)-c>g-1$. Together with Lemma 3.1, this implies that $g\left(X^{(c)}\right)=g+c$.

Case $2 \Sigma$ is weakly reducible.

In [27] Sedgwick proved a relative version of Casson and Gordon's seminal theorem [1], proving that an appropriately chosen weak reduction of a minimal genus Heegaard surface yields an essential surface (see the statement and the proof of Theorem 1.1 of [27], cf [14, Theorem 3.1]). Denote by $\widehat{F}$ the essential surface obtained by weakly 
reducing $\Sigma$. Let $F$ be a connected component of $\widehat{F}$. Since $F \subset X^{(c)} \subset M$, it separates. Hence by [9, Proposition 2.13], $\Sigma$ weakly reduces to $F$. Note that $\chi(F) \geq \chi(\Sigma)+4$.

Claim $F$ can be isotoped into $Q^{(c)}$.

Proof of Claim Recall the definitions of $T, X^{\prime}$ and $Q^{(c)}$ from the proof of Proposition 2.2. Assume, for a contradiction, that $F$ cannot be isotoped into $Q^{(c)}$. Since $X$ does not admit an essential surface $S$ with $\chi(S) \geq 4-2(g+c), X$ is irreducible. Minimize $|F \cap T|$. Since $F$ and $T$ are essential and $X$ and $Q^{(c)}$ are irreducible, $F \cap T$ consists of a (possibly empty) collection of curves that are essential in both surfaces. If $F \cap X^{\prime}$ compresses, then, since the curves of $F \cap T$ are essential in $F$, so does $F$, contradiction. Since $T$ is a torus, boundary compression of $F \cap X^{\prime}$ implies a compression (see, for example, [8, Lemma 2.7]). Finally, minimality of $|F \cap T|$ implies that no component of $F \cap X^{\prime}$ is boundary parallel. Thus, every component of $F \cap X^{\prime}$ is essential (including the case $\left.F \subset X^{\prime}\right)$. Since no component of $F \cap Q^{(c)}$ is a disk or a sphere, $\chi\left(F \cap X^{\prime}\right) \geq \chi(F) \geq \chi(\Sigma)+4$. By Lemma 3.1, $\chi(\Sigma) \geq 2-2(g+c)$, thus $\chi(\Sigma)+4 \geq 6-2(g+c)$. Hence $\chi\left(F \cap X^{\prime}\right) \geq 6-2(g+c)$. Since $X^{\prime} \cong X$, this contradicts the assumption of Proposition 3.2. This proves the claim.

Since $F$ is a closed incompressible surface in $Q^{(c)}$, and $Q^{(c)}$ is a punctured annulus cross $S^{1}, F$ is a vertical torus (see, for example, [4, VI.34]).

First, suppose that $F$ is not boundary parallel in $Q^{(c)}$. Then $F$ decomposes $X^{(c)}$ as $X^{(p+1)} \cup_{F} D(c-p)$, where $0 \leq p \leq c$ is an integer and $D(c-p)$ is a disk with $c-p$ holes cross $S^{1}$. Note that since $F$ is not parallel to a component of $\partial Q^{(c)}, c-p \geq 2$. Therefore $p+1<c$. This, together with the assumption of the proposition, implies that $X$ does not admit an essential surface $S$ with $\chi(S) \geq 4-2(g+(p+1))$, and that $K$ does not admit a $(g-1, p)$ position; hence the induction hypothesis applies to $X^{(p+1)}$, giving that $g\left(X^{(p+1)}\right)=g+p+1$. By Schultens [25], $g(D(c-p))=c-p$. Since $F$ was obtained by weakly reducing a minimal genus Heegaard surface [9, Proposition 2.9] (see also [25, Remark 2.7]) gives:

$$
\begin{aligned}
g\left(X^{(c)}\right) & =g\left(X^{(p+1)}\right)+g(D(c-p))-g(F) \\
& =(g+p+1)+(c-p)-1 \\
& =g+c .
\end{aligned}
$$

Next, suppose that $F$ is boundary parallel in $Q^{(c)}$. Since $F$ is essential in $X^{(c)}$, it cannot be isotopic to a component of $\partial X^{(c)}$ and must therefore be isotopic to $\partial Q^{(c)} \backslash \partial X^{(c)}=T$. This gives the decomposition $X^{(c)}=X^{\prime} \cup_{F} Q^{(c)}$. Since $X^{\prime} \cong X$, 


$$
\begin{aligned}
& g\left(X^{\prime}\right)=g \text {. By [25] } g\left(Q^{(c)}\right)=c+1 \text {. We get, as above: } \\
& g\left(X^{(c)}\right)=g\left(X^{\prime}\right)+g\left(Q^{(c)}\right)-g(F) \\
& =g+(c+1)-1 \\
& =g+c \text {. }
\end{aligned}
$$

This completes the proof of Proposition 3.2.

Proposition 3.3 Let $m \geq 1$ and $c \geq 0$ be integers, and let $\left\{K_{i} \subset M_{i}\right\}_{i=1}^{m}$ be knots in closed orientable manifolds. Suppose that $M_{i}$ does not admit a nonseparating surface $(1 \leq i \leq m)$. Denote the exterior of $K_{i}$ by $X_{i}$, and the exterior of $\#_{i=1}^{m} K_{i}$ by $X$. Let $g$ be an integer so that $g\left(X_{i}\right) \leq g(1 \leq i \leq m)$.

Suppose that no $X_{i}$ admits an essential surface $S$ with $\chi(S) \geq 4-2 g(m+c)$, and that no $K_{i}$ admit a $\left(g\left(X_{i}\right)-1, m+c-1\right)$ position. Then we have:

$$
g\left(X^{(c)}\right)=\sum_{i=1}^{m} g\left(X_{i}\right)+c .
$$

Proof Suppose first that $m=1$. Note that $4-2 g(1+c) \leq 4-2(c+g)$; therefore Proposition 3.3 follows from Proposition 3.2 in this case. Assume from now on $m \geq 2$.

We induct on $(m, c)$ ordered lexicographically, where $m$ is the number of summands and $c$ is the number of curves drilled. Note that by Miyazaki [12], $m$ is well defined (see [9, Claim 1]).

By Lemma 3.1, Inequality (1) in Section 1, and the assumption that $g\left(X_{i}\right) \leq g$ for all $i$, we get: $g\left(X^{(c)}\right) \leq g(X)+c \leq \sum_{i=1}^{m} g\left(X_{i}\right)+c \leq m g+c$. Since $g \geq 2$, we have that $g\left(X^{(c)}\right) \leq g(m+c)$.

By assumption, for all $i, X_{i}$ does not admit an essential surface $S$ with $\chi(S) \geq$ $4-2 g(m+c)$. Hence by the Swallow Follow Torus Theorem [9, Theorem 4.1], any minimal genus Heegaard surface for $X^{(c)}$ weakly reduces to a swallow follow torus $F$ giving the decomposition $X^{(c)}=X_{I}^{\left(c_{1}\right)} \cup_{F} X_{J}^{\left(c_{2}\right)}$, where $I \subset\{1, \ldots, m\}$, $K_{I}=\#_{i \in I} K_{i}, K_{J}=\#_{i \notin I} K_{i}, X_{I}=E\left(K_{I}\right), X_{J}=E\left(K_{J}\right)$, and $c_{1}+c_{2}=c+1$ (for details see the first paragraph of Section 4 of [9]). Denote the number of factors of $K_{I}$, $|I|$, by $m_{1}$, and the number of factors of $K_{J}, m-|I|$, by $m_{2}$. Note that $m_{1}=0$ or $m_{2}=0$ are possible. However, at least one of $m_{1}$ or $m_{2}$ is not zero so by symmetry we may assume $m_{1} \neq 0$.

First assume that $m_{1}=m$. Then $m_{2}=0$ and $X_{J}^{\left(c_{2}\right)}$ is a disk with $c_{2}$ holes cross $S^{1}$. Since $F$ is essential [27, Theorem 1.1], $c_{2} \geq 2$. Then $c_{1}=c-c_{2}+1 \leq c-1$. Since $m_{1}=m$, we see that $m_{1}+c_{1} \leq c+m-1$. By assumption, no $X_{i}(1 \leq i \leq m)$ admits 
an essential surface $S$ with $\chi(S) \geq 4-2 g\left(m_{1}+c_{1}\right)>4-2 g(m+c)$. Hence, the induction hypotheses applies to $X_{I}^{\left(c_{1}\right)} \cong X^{\left(c_{1}\right)}$, showing that

$$
g\left(X_{I}^{\left(c_{1}\right)}\right)=\sum_{i=1}^{m} g\left(X_{i}\right)+c_{1} .
$$

Since $X_{J}^{\left(c_{2}\right)}$ is homeomorphic to a disk with $c_{2}$ holes cross $S^{1}, g\left(X_{J}^{\left(c_{2}\right)}\right)=c_{2}$ by [25]. Since $F$ was obtained by weakly reducing a minimal genus Heegaard surface, Proposition 2.9 of [9] and the fact that $c_{1}+c_{2}=c+1$, we get:

$$
\begin{aligned}
g\left(X^{(c)}\right) & =g\left(X_{I}^{\left(c_{1}\right)}\right)+g\left(X_{J}^{\left(c_{2}\right)}\right)-g(F) \\
& =\left(\sum_{i=1}^{m} g\left(X_{i}\right)+c_{1}\right)+c_{2}-1 \\
& =\sum_{i=1}^{m} g\left(X_{i}\right)+c .
\end{aligned}
$$

This proves Proposition 3.3 when $m_{1}=m$.

Next assume that $m_{1}<m$. By assumption $m_{1}>0$, hence $m_{2}<m$. By construction $c_{1} \leq c+1$, and $c_{2} \leq c+1$. Hence $m_{1}+c_{1} \leq m+c$, and $m_{2}+c_{2} \leq m+c$. By assumption, no $X_{i}(1 \leq i \leq m)$ admits an essential surface $S$ with $\chi(S) \geq 4-2\left(m_{j}+c_{j}\right) g \geq$ $4-2(m+c) g(j=1,2)$. Hence the induction hypothesis applies to $X_{I}^{\left(c_{1}\right)}$ and $X_{J}^{\left(c_{2}\right)}$, giving $g\left(X_{I}^{\left(c_{1}\right)}\right)=\sum_{i \in I} g\left(X_{i}\right)+c_{1}$, and $g\left(X_{J}^{\left(c_{2}\right)}\right)=\sum_{i \notin I} g\left(X_{i}\right)+c_{2}$. We get, as above:

$$
\begin{aligned}
g\left(X^{(c)}\right) & =g\left(X_{I}^{\left(c_{1}\right)}\right)+g\left(X_{J}^{\left(c_{2}\right)}\right)-g(F) \\
& =\left(\sum_{i \in I} g\left(X_{i}\right)+c_{1}\right)+\left(\sum_{i \notin I} g\left(X_{i}\right)+c_{2}\right)-1 \\
& =\sum_{i=1}^{m} g\left(X_{i}\right)+c_{1}+c_{2}-1 \\
& =\sum_{i=1}^{m} g\left(X_{i}\right)+c .
\end{aligned}
$$

This completes the proof of Proposition 3.3.

Remark 3.4 For $m \geq 2$, the proof is an application of the Swallow Follow Torus Theorem [9, Theorem 4.1]. In [9, Remark 4.2] it was shown by means of a counterexample that the Swallow Follow Torus Theorem does not apply to $X^{(c)}$ when $m=1$. Hence the argument of the proof of Proposition 3.3 cannot be used to simplify the proof of Proposition 3.2.

\section{Proof of Theorem 1.2}

Fix $g \geq 2$ and $n \geq 1$. Let $\mathcal{K}_{g, n}$ be the set of all knots $K \subset S^{3}$ with the following three properties:

(a) $g(E(K)) \leq g$. 
(b) $K$ does not admit a $(t(K), n)$ position.

(c) $E(K)$ does not admits an essential surface $S$ with $\chi(S) \geq 4-2 g n$.

Fix $h$ satisfying $2 \leq h \leq g$. There exist infinitely many knots in $S^{3}$, each admitting a genus $h$ Heegaard splitting of distance greater than $\max \{2 g n-2,2(h+n-1)\}$, by [11, Theorem 3.1]. Let $K_{h}$ be such a knot, and $X_{h}$ its exterior.

Since $X_{h}$ admits a genus $h$ Heegaard splitting with distance greater than $2(h+n-1) \geq$ $2 h$ (as $n \geq 1$ ), by [24, Corollary 4.7] this splitting must be minimal genus; in particular, $g\left(E\left(K_{h}\right)\right)=h$. Since $X_{h}$ admits a Heegaard splitting with distance greater than $2(h+n-1)$, by Proposition 2.6, $K_{h}$ does not admit a $(h-1, n)=(t(K), n)$ position. Since $X_{h}$ admits a Heegaard splitting with distance greater than $2 g n-2$, by Proposition 2.5, $X_{h}$ does not admits an essential surface $S$ with $\chi(S) \geq 4-2 g n$. We see that $K_{h} \in \mathcal{K}_{g, n}$ and hence $\mathcal{K}_{g, n}$ contains infinitely many knots $K$ with $g(X)=h$. This proves that $\mathcal{K}_{g, n}$ fulfills Conclusion (1) of Theorem 1.2.

Since (for any $\left.K \in \mathcal{K}_{g, n}\right) X$ does not admit an essential surface $S$ with $\chi(S) \geq 4-2 g n$, and $K$ does not admit a $(t(K), n)$ position, applying Proposition 3.3 with $m \leq n$ and $c=0$, we see that the knots in $\mathcal{K}_{g, n}$ fulfill Conclusion (2) of Theorem 1.2.

By [10, Theorem 1.2] for any knot $K^{\prime} \subset S^{3}$, there exists $N$ so that if $n>N$, then $g\left(E\left(n K^{\prime}\right)\right)<n g\left(E\left(K^{\prime}\right)\right)$. This shows that $K^{\prime} \notin \mathcal{K}_{g, n}$ for $n>N$. Hence $K^{\prime} \notin \bigcap_{n=1}^{\infty} \mathcal{K}_{g, n}$. As $K^{\prime}$ was arbitrary, $\bigcap_{n=1}^{\infty} \mathcal{K}_{g, n}=\varnothing$.

This completes the proof of Theorem 1.2.

\section{References}

[1] A J Casson, C M Gordon, Reducing Heegaard splittings, Topology Appl. 27 (1987) 275-283 MR918537

[2] K Hartshorn, Heegaard splittings of Haken manifolds have bounded distance, Pacific J. Math. 204 (2002) 61-75 MR1905192

[3] J Hempel, 3-manifolds as viewed from the curve complex, Topology 40 (2001) 631657 MR1838999

[4] W Jaco, Lectures on three-manifold topology, CBMS Regional Conference Series in Math. 43, Amer. Math. Soc. (1980) MR565450

[5] J Johnson, Bridge number and the curve complex arXiv:math.GT/0603102

[6] J Johnson, A Thompson, On tunnel number one knots which are not $(1, n)$ arXiv: math. GT/0606226v3 
[7] T Kobayashi, Y Rieck, Knots with $g(E(K))=2$ and $g(E(3 K))=6$ and Morimoto's Conjecture, to appear in Topology Appl. (special volume dedicated to Yves Mathieu and Michel Domergue) arXiv:math.GT/0701766

[8] T Kobayashi, Y Rieck, Local detection of strongly irreducible Heegaard splittings via knot exteriors, Topology Appl. 138 (2004) 239-251 MR2035483

[9] T Kobayashi, Y Rieck, Heegaard genus of the connected sum of m-small knots, Comm. Anal. Geom. 14 (2006) 1037-1077 MR2287154

[10] T Kobayashi, Y Rieck, On the growth rate of the tunnel number of knots, J. Reine Angew. Math. 592 (2006) 63-78 MR2222730

[11] Y N Minsky, Y Moriah, S Schleimer, High distance knots, Algebr. Geom. Topol. 7 (2007) 1471-1483 MR2366166

[12] K Miyazaki, Conjugation and the prime decomposition of knots in closed, oriented 3-manifolds, Trans. Amer. Math. Soc. 313 (1989) 785-804 MR997679

[13] Y Moriah, Heegaard splittings of knot exteriors arXiv:math.GT/0608137

[14] Y Moriah, On boundary primitive manifolds and a theorem of Casson-Gordon, Topology Appl. 125 (2002) 571-579 MR1935173

[15] Y Moriah, H Rubinstein, Heegaard structures of negatively curved 3-manifolds, Comm. Anal. Geom. 5 (1997) 375-412 MR1487722

[16] Y Moriah, E Sedgwick, The Heegaard structure of Dehn filled manifolds arXiv: math.GT/07061927v1

[17] K Morimoto, On the super additivity of tunnel number of knots, Math. Ann. 317 (2000) 489-508 MR1776114

[18] K Morimoto, M Sakuma, Y Yokota, Examples of tunnel number one knots which have the property " $1+1=3$ ", Math. Proc. Cambridge Philos. Soc. 119 (1996) 113-118 MR1356163

[19] Y Rieck, Heegaard structures of manifolds in the Dehn filling space, Topology 39 (2000) 619-641 MR1746912

[20] Y Rieck, E Sedgwick, Finiteness results for Heegaard surfaces in surgered manifolds, Comm. Anal. Geom. 9 (2001) 351-367 MR1846207

[21] Y Rieck, E Sedgwick, Persistence of Heegaard structures under Dehn filling, Topology Appl. 109 (2001) 41-53 MR1804562

[22] M Scharlemann, Proximity in the curve complex: boundary reduction and bicompressible surfaces, Pacific J. Math. 228 (2006) 325-348 MR2274524

[23] M Scharlemann, J Schultens, Comparing Heegaard and JSJ structures of orientable 3-manifolds, Trans. Amer. Math. Soc. 353 (2001) 557-584 MR1804508

[24] M Scharlemann, M Tomova, Alternate Heegaard genus bounds distance, Geom. Topol. 10 (2006) 593-617 MR2224466 
[25] J Schultens, The classification of Heegaard splittings for (compact orientable surface) $\times S^{1}$, Proc. London Math. Soc. (3) 67 (1993) 425-448 MR1226608

[26] J Schultens, Additivity of tunnel number for small knots, Comment. Math. Helv. 75 (2000) 353-367 MR1793793

[27] E Sedgwick, Genus two 3-manifolds are built from handle number one pieces, Algebr. Geom. Topol. 1 (2001) 763-790 MR1875617

[28] M Tomova, Distance of Heegaard splittings of knot complements arXiv: math.GT/0703474v2

Department of Mathematics, Nara Women's University

Kitauoya-Nishimachi, Nara, 630-8506, Japan

Department of Mathematical Sciences, University of Arkansas

Fayetteville, AR 72701

tsuyoshi@cc.nara-wu.ac.jp, yoav@uark.edu

Received: 1 May 2007 Revised: 24 April 2008 